\title{
COVID-19: An Urgency to Address Mental Health Consequences
}

\author{
Vito Zepinic \\ PsychClinic P/L, London, UK \\ Email: vito@psychclinic.net
}

How to cite this paper: Zepinic, V. (2021). COVID-19: An Urgency to Address Mental Health Consequences. Psychology, 12, 735748

https://doi.org/10.4236/psych.2021.125045

Received: April 15, 2021

Accepted: May 23, 2021

Published: May 26, 2021

Copyright (c) 2021 by author(s) and Scientific Research Publishing Inc. This work is licensed under the Creative Commons Attribution International License (CC BY 4.0).

http://creativecommons.org/licenses/by/4.0/

\begin{abstract}
The novel COVID-19 pandemic has swept the entire world, seriously threatening the safety of all mankind, causing psychosocial panic and global financial crisis. Public measures taken to reduce the spread of Coronavirus have disrupted social and economic systems. At the same time, the entire world population is exposed to various persistent stressors that lead to mental health problems. These include social isolation, loss of employment, severe stress due to loved ones being infected or deceased, and fear of being affected by an invisible enemy. Given the psychological, social and economic burden placed on the entire world's population, the impact on mental health is still not fully graspable, as the COVID-19 pandemic is one continuum of traumatic event. When facing stressful situations, most individuals may cope in a resilient manner; however, it is common that resilience decreases due to uncertainty and continuation of the stressor. Thereby, the strategies in dealing with the pandemic's impact upon mental health problems require a long-term concept.
\end{abstract}

\section{Keywords}

COVID-19, Pandemic, Quarantine, Mental Health, Stress-Related Disorders

\section{Introduction}

It is well-known that infectious diseases historically have been, and remain, frightening and cause a psychological impact upon individuals due to the risk factors for contamination. In case of an epidemic, affected patients and health care workers experience fears of the illness and fears of contagion to family and friends. The public fear of a highly contagious virus, similar to other life-threatening diseases, usually changes people's daily life routine and creates a psychosocial impact (i.e., anxiety, depression, stress-related disturbances, social isolation, sub- 
stance abuse, stigma, occupational problems, financial difficulties). It is common that, due to its quick spread, a highly contagious virus causes long-term consequences on people's mental health (Bialer \& Wallack, 2000). For example, rates of depression in HIV infected patients range up to $35 \%$, with a comorbid risk of the suicidal behaviours (Stober et al., 1997).

A novel invisible enemy in the form of Coronavirus disease (COVID-19), firstly detected in China in December 2019, quickly spread worldwide, creating a global pandemic. Without proper prevention (in the beginning of spring 2020), the rapidly spreading pandemic turned Europe (in particular Italy) into one of the epicentres of the outbreak. In general, most health systems were unprepared to deal with COVID-19, nor had enough knowledge about the nature of Coronavirus.

Thus, the World Health Organisation (WHO, 2020) advised a global health emergency considering the rapid, and without control, spreading of COVID-19. However, the warning was not taken seriously enough to stop spreading of the virus; and some countries (i.e., USA) accused WHO of being politicised by its member(s). Moreover, the USA administration withdrew further financial support to $\mathrm{WHO}$, what show how the world is divided instead of being strongly united in dealing with the world's common enemy.

The crisis of COVID-19 was often referred as a "marathon, not a sprint", which depicts the uncertainty when dealing with the virus spreading, control, and the possibility of it becoming a recurrent seasonal infection like any other virus. Various medical authorities and experts have described the COVID-19 situation as unprecedented, not only because of the virus's contagiousness, speed and scale of the outbreak, but also of the preventive measures which negatively impact on other important life domains.

A global wakeup appeared to some extent in early March 2020 when the Italian Government imposed lockdown measures on the national territory-people were asked to stay at home and socially isolate themselves to prevent further contagions. Thus, the rapid escalation of COVID-19 urged many countries to increase efforts to control spread of the disease. On 11 March 2020, the WHO declared that the COVID-19 outbreak is a global pandemic (WHO, 2020). Due to a high contagious level of the Coronavirus and lockdown measures to mitigate its spread, people's daily life around the world changed drastically. It has been previously shown (Brooks et al., 2018; Tucci et al., 2017; Allan et al., 2020) that health emergencies during epidemics and pandemics, such as SARS, MERS, ZIKA, and Ebola disease, lead to serious detrimental and long-lasting psychosocial consequences.

Quarantined and/or self-isolated individuals may struggle with loneliness, stigmatisation, and isolation. Both individual and group reactions to real or imagined threats of infectious diseases also may include hysterical and phobic behaviours. Anxiety about acquiring a feared disease may lead to conversion symptoms, hypochondriacal preoccupation, and unnecessary avoidance beha- 
viour (Levenson \& Schneider, 2005).

Major depression and stress-related disturbances are a risk factor for individuals, who suffer due to isolation, fears, loneliness, loss of trust, and uncertainty, and may cause change in behaviour, intensification of the substance abuse, and an exacerbation of individuals' self-destructive behaviours. Distress symptoms in the individuals have been found to correlate with poor performances, decreased global quality of life, binge eating, lowered level of tolerance, frustration and aggressiveness, loss of self-coherence and continuity (Zepinic, 2017).

Suppose we calculate traumatic events due to COVID-19 (i.e., death of a spouse or close family member, separation from others, loss of employment, change in health of family member, death of a close friend, change in finances, etc.) from the Social Readjustment Rating Scale (Holmes, 1978), we can find that many individuals will score over 300 points, which indicates detrimental effects of cumulative stress. According to the multilevel framework of a pandemic's impact, COVID-19 includes risk factors and stressor on the individual's level by two issues: biological (i.e., having COVID-19) and psychosocial (i.e., the fear of contracting Coronavirus, perceived lack of support, death of loved ones, a severe COVID-19 infection of loved ones, working in health care).

Evidently, psychopathology and psychological distress are high during the pandemic (COVID-19), which may make the trauma-syndrome construct: interpersonal sensitivity, depression, anxiety, hostility, phobic-anxiety, altered selfconcept, problems in affect regulations, and impaired interpersonal relations (Zepinic, 2019). Corresponding with this is the fact that COVID-19 causes a prolonged trauma experience (usually under prolonged and repeated lockdown conditions or self-isolation) with severe uncertainty and loss of meaning.

\section{Is COVID-19 Impact on Mental Health Undervalued?}

A central task of the psychological approach to medically sick individuals is to understand patients' subjective experiences of sustained illness to design therapeutic interventions that modulate the patients' behavioural or emotional responses, decrease their distress, and improve their medical condition (Groves \& Muskin, 2005). In outpatient or inpatient practice, mental health professionals (i.e., psychiatrists or psychologists) face tremendous diversity of emotional and behaviour responses to physical diseases.

Some patients or those who are dealing with loved one's disease, or who are exposed to the epidemic or pandemic, have difficulties in overcoming intense emotions, such as sadness, hopelessness, fear, loneliness, desperation, and inferiority. Also, patients may experience insecurity, neediness, pessimism, uncertainty, vulnerability, impatience, and worries. Thus, the concept of stress has been central in the development of psychosomatic theory of the illness involving stress paradigms relevant to the psychosomatic medicine.

The events, such as the COVID-19 pandemic, are deemed stressful as individuals perceive them as threatening to life, well-being, and emotional or psy- 
chosocial security. The capacity to adapt to the threatening events (i.e., familial discord, emotional deprivation, goal frustration, object loss, separation, social isolation, and unemployment) determines the nature and severity of psychophysiological response patterns (Zepinic, 2019).

We may observe the COVID-19 impact on people's mental health within three phases: 1) the alarm reactions; 2 ) the stage of resistance, in which adaptation is achieved; and 3) the stage of exhaustion, in which acquired adaptation may be lost. Also, numerous studies in the area of psychoendocrinology demonstrate that in the experimental stress situations, physiological reactions are largely influenced by the emotional response to the stimuli and not solely determined by the nature of the stimulus itself.

There are numerous studies and clinical observations in the fields of psychiatry and clinical psychology which attempt to explain a tremendous variety of the individual responses to the stressors of illness and to account for these individual differences. The concept of stress caused by COVID-19, personality types, coping strategies, and defence mechanisms can be integrated into a framework that illustrates the complexity of people's responses to the Coronavirus.

This framework should be holotropic as an attempt to integrate the psychodynamic concepts of individuals' character style and intrapsychic defences with other psychological concepts (Zepinic, 2021). The importance of individual subjectivity shall be emphasised through the placement of coping styles, defence mechanisms, personality styles, and the appraised meaning of the COVID-19 pandemic as central mediators of the behavioural and emotional responses.

The COVID-19 pandemic represents a life-threatening traumatic event, as it can lead to hospitalisation and even death. Several studies revealed that comorbidity of mental health disorders (stress-related disorders, depression, anxiety) due to Coronavirus is very high. Due to the imposed extensive worldwide restriction (i.e., limits on freedom of movement, closure of the nonessential businesses, requirement to stay at home or self-isolate, restrict social contacts to slow the spread of a contagion) exposed people to severe psychological distress. In general, we can emphasise that lockdown due to COVID-19 is a highly stressful event with severe mental health outcomes.

For example, in survey of 1041 general population during the lockdown in the United Kingdom and the Republic of Ireland (Karatzias et al., 2020), using the International Trauma Questionnaire (Cloitre et al., 2019), it was found that 17.7\% of the participants met the diagnostic requirements for the COVID-19 related PTSD. Among the participants who screened for the PTSD, 53.8\% met criteria for depression; $49.5 \%$ met the criteria for generalised anxiety disorder; and $60.3 \%$ met criteria for either anxiety or depression. The study found that stress-based psychopathology was associated with people of younger age, those who are living with children, living in a city, having an existing chronic health problem, or higher levels of perceived vulnerability to COVID-19.

In another study (Hyland et al., 2020), it was found that a substantial proportion of the population (23\%) screened positive for depression, $20 \%$ screened for 
a generalised anxiety, and $28 \%$ for mixed anxiety/depression. The research also found that these conditions were associated with younger age, female sex, loss of income due to the COVID-19 infection. Simultaneously, a study in Spain (Rodriguez-Rey et al., 2020) found that nearly 37\% participants showed psychological distress (PTSD) due to the COVID-19 pandemic, and the estimated impact was consistently higher for women and younger participants.

Most studies about trauma-spectrum symptoms of COVID-19 involved the general population (Karatzias et al., 2020; Hyland et al., 2020; Rodriguez-Rey et al., 2020; Rossi et al., 2021), which appeared under a high risk of suffering from stress-related disorders, depression, and anxiety during the lockdown. In essence, the COVID-19 risk factors upon people's mental health could be summarise as follow: first, the lockdown status imposed upon the entire population in particular country or area, mandatory being under quarantine or self-isolated had a significant impact on people's mental health.

Since the COVID-19 pandemic commenced, these measures have been introduced in many countries that lasted for few months. During these measures some individuals have been separated from loved ones for several months and felt concerns for the welfare of their loved ones. Additionally, people under the lockdown feel isolated, insecure, lonely, melancholic, trapped and vulnerable with increased levels of depression, anxiety, and desperation. Most people in quarantine, or those being self-isolated, may show full scale of the emotion-related risk factors, including anxiety sensitivity, emotional reactivity, and distress tolerance, which are predictable for disturbances on one's mental health.

Lockdowns also caused loss of employment or changes in a working activity for which employees had not been prepared because the lockdown measures have mostly been imposed immediately. Some working activities and employments were discontinued which some employees saw similar as being fired at work.

Study in Italy (Rossi et al., 2021) reports different (multiple) mental health outcomes on general population due to lockdown: $23 \%$ reported symptoms of adjustment disorder, 37\% reported PTSD, 22\% high perceived stress, $21 \%$ anxiety, and $17 \%$ depression. In the survey based on self-report assessment, the majority of respondents $(75.8 \%, n=1320)$ indicated that the COVID-19 pandemic was a stressful event for them. It was also revealed that being female and younger was associated with all of the mental health outcomes despite the fact that most deaths occurred in the older generation.

The second risk factor of the COVID-19 pandemic for disturbances of people's mental health is with those individuals who were infected, hospitalised, of whom loved one is infected or deceased due to COVID-19. Any illness and hospitalisation of a patient or their loved ones is a stressful event. These conditions may produce stressor-related disorders (i.e., reactive attachment disorder, disinhibited social engagement disorder, PTSD or acute stress disorder, adjustment disorder, depression). 
According to DSM-5 (APA, 2013), the PTSD diagnostic Criterion $A$ fits to those individuals who are hospitalised or infected by COVID-19 and are being treated in self-isolation, or whom loved one is infected, hospitalised or deceased. They are directly or indirectly experiencing the traumatic event(s): witnessing in person the event as occurred to others, or learning that the traumatic event occurred to a close family member or friend. They also experiencing repeated or extreme exposure to aversive details of the traumatic event (the COVID-19 pandemic).

Studies (Karatzias et al., 2020; Rossi et al., 2021) also found that, due to COVID19, alongside the classic PTSD core symptoms (hyperarousal, re-experiencing, and avoidance), people also suffer the Disturbances in Self-Organisation (DSO): 1) problems in one's affect regulation (i.e., heightened or lowered emotional reactivity; feelings of neediness, dejection, hopeless), 2) altered self-concept (i.e., feelings like failure or worthlessness; incompetent, inadequate; self-conscious; trapped), and 3) impaired interpersonal relations (i.e., difficulties in establishing or sustaining interpersonal connections, or avoidance of relationships; isolated; dejected or rejected; ignored) which satisfy definition of the complex-PTSD in ICD-11 (Cloitre et al., 2019; WHO, 2018).

Affect regulation is a multidimensional construct involving: 1) awareness, understanding, and acceptance of the emotions; 2) ability to control impulsive and self-harm behaviours when experiencing negative emotions; 3) flexible use of situationally proper strategies to modulate and control the intensity and duration of emotional responses; and 4) volition to experience negative emotions in the pursuit of meaningful activities (Zepinic, 2019). Affect dysregulation (as it is defined in ICD-11) would be characterised by deficits in one or more of these abilities.

Extended or frequent experiences of distressing emotions during the COVID-19 pandemic may also lead individual to engage in a risky and/or self-destructive behaviour in effort to avoid or to find "way out" from unwanted emotional experiences. Numerous studies provided evidence that the specific maladaptive emotion regulation strategies (i.e., dissociation, difficulties using cognitive reappraisal, avoidance and rumination) may predict development of the stress-related disorders due to repeated or prolonged stressful condition.

Complex PTSD, also called Complex Trauma Syndrome (Cloitre et al., 2019; Courtois \& Ford, 2009; Herman, 1992; Zepinic 2019, 2021), is a new category in the ICD-11 being distinct from its PTSD-sibling, and other stress-related disorders. Studies (Cloitre et al., 2019; Herman, 1992; Ford et al., 2005; Zepinic, 2001, 2021) found that increased depression, evidence of the suicidal behaviours, dissociative symptoms, as well as unspecific psychopathological symptoms (i.e., sleep problems, eating disorders, other psychological problems) also characterise Complex Trauma Syndrome (complex PTSD). In general, Complex Trauma Syndrome highlighting the trauma impact of prolonged and/or repetitive interpersonal stress upon the trauma victim's self independently of PTSD standard 
triad cluster symptoms (hyperarousal, re-experience, and avoidance) (Zepinic, 2017, 2021).

According to ICD-11, the complex-PTSD retains the core triad clusters including: 1) avoidance of internal and external trauma reminders related to the event(s); 2) re-experiencing the traumatic event(s) in the form of the flashbacks or nightmares; and 3) persistent-heightened sense of current threat, indicated by the hypervigilance or increased startle response (WHO, 2018; Zepinic, 2019, 2021). However, with added disturbances in self-regulation clusters, the ICD-11 taxonomic structure means that an individual can only be diagnosed with PTSD or complex-PTSD, not both.

On the other hand, the DSM-5 (APA, 2013) has taken a different approach for addressing complexity in PTSD condition by expanding its previous diagnostic criteria to include "negative alterations in cognition and mood", which overlaps with the ICD-11 complex-PTSD's DOS clusters (negative self-concept, affective dysregulation, and difficulties in relationships). In addition, the DSM-5 for the first-time expanded criteria for a specifier of a dissociative subtype (depersonalisation and derealisation) which is commonly associated with a complex presentation of PTSD.

Thus, two nosological systems (DSM-5 and ICD-11) differ whether or not the complex-PTSD constitutes a distinct diagnostic category. Our opinion is that ICD-11 diagnosis has aimed to be more specific regarding complex-PTSD and it is both conceptually and clinically useful as a distinct entity which is highly prevalent in survivors of prolonged and/or repeated trauma and have significantly higher levels of dissociative experiences compared to those with simple PTSD (Zepinic, 2019, 2021).

The Complex Trauma Syndrome (complex PTSD) is also associated with high levels of the cumulative trauma exposure and clinically represents a distinct construct. The syndrome is usually characterised by intense emotional pain, persistent and pervasive preoccupation with the trauma event(s), feeling one has lost a part of one's self, emotional numbness, bitterness or anger, maladaptive appraisals about the self, an inability to experience positive mood and feelings that life is empty or meaningless (Zepinic, 2019).

Traumatic event(s), such as COVID-19, affects all structures of the individual's self-one's image of the body; the internalised images of others; and one's values and ideals-and leads to a sense that self-coherence and one's goals are invaded, assaulted and systematically broken down (Zepinic, 2017, 2019). Traumatic event(s) overwhelms the ordinary human adaptations to life and generally involve threats to life or bodily integrity. The most affected by the traumatic event(s) are those individuals with vulnerable self-structure and weak defence mechanisms.

Trauma impacts the psychic core of the soul of the victim and generates a search for meaning as to why the event had to happen. The vulnerable self-structure may be seen in following ways (Zepinic, 2019): 
1) difficulties in self-regulation, such as: self-esteem maintenance, affect tolerance, and the sense of self-continuity, or sense of personal agency. Such difficulties with self-regulation, as in "developmental arrest", can result in addictive behaviour (alcohol or illicit drugs), or compulsive activities;

2) the appearance of specific clinical symptoms, such as: frequent upsurges of anxiety (fears), depression, or irritability; and specific fears or phobias regarding the external world or one's own bodily integrity (i.e., being quarantined or hospitalised due to COVID-19); and

3) reliance on primitive or less-developed forms of one's self-object relatedness with attachment figures.

Life-threatening event(s), such as COVID-19, may lead to de-centering of one's self, loss of groundedness and a sense of sameness, continuity and ego-fragility, leaving scars on one's "inner agency" of the psyche (Zepinic, 2019). Fragmentation of self-identity has consequences for the individual's psychological stability, well-being, and psychic integration, resulting in proneness to dissociation. In many cases the fragmentation of self-identity is a fracture of the person's soul and spirit, like a broken connection in one's essential sense of meaning and existence.

Clinicians (Cloitre et al., 2019; Herman, 1992; Ford et al., 2005; Zepinic, 2019) are agreed that complex trauma is analogous to a high velocity bullet piercing through the body, tearing apart internal organs critical to survival. Similarly, whereas the brain and heart are critical organs of the body, the self-continuity and cohesion are the core psychic issues for psychological functioning. As the brain and heart are essential structures with interrelated functions, the wholeness of the self is dynamically crucial for one's mental well-being (Zepinic, 2019). Without meaningful ways to understand traumatic damage to the one's self by the complex traumatic event(s), it would be like trying to understand one's degenerative neurological disorder(s) without understanding how the brain functions.

One of issues in understanding COVID-19 impact upon people's psychosocial life is to understand the development of their "survival syndrome"-accustomed behaviour and relatedness to the danger of being infected by Coronavirus. In a pathogenic environment such as the COVID-19 pandemic, the relatedness and attachment are disturbed and, instead of bringing psychic gain and equilibrium back, the post-pandemic will probably bring the aversive results (challenges in the ordinary relationships) - the core affective phenomena encountered alongside disruptive responses to attachment figures (Zepinic, 2015).

Disrupted attachment ties and compromise self-integrity may lead people into an unbearable emotional state: experience of helplessness, loneliness, confusion, emptiness, and despair. In the post-pandemic time, the people's relatedness, dynamics, and aliveness may still be based on the "survival skills" acquired during the COVID-19 pandemic, including fear of both abandonment and closeness, and affect dysregulation, mediated the relationship between individual and oth- 
ers. Knowledge about the dangerousness of the outer world and one's incompetence of the self may influence how the traumatic memory was encoded and how post-pandemic experiences are perceived.

Thus, complex interconnections may appear to be involved both within and between the constructs of the stress syndrome instead of social adaptation. Detachment is thought to arise from intense fear or trauma of being contaminated by COVID-19, and this condition may lead to an altered state of consciousness involving a sense of disconnection from the outer world. The pandemic survivors' narratives have been characterised as fragmented and disorganised as the result of several mechanisms known to interfered with the processing of information encoded under intense distress.

Due to negative social appraisals of the lockdowns (particularly in Europe), it is expected that similar measures for mitigation of the COVID-19 pandemic would carry further adverse social and psychological consequences. The public's intrusive and deliberate rumination in relation to such measures is because they decrease the social support which is an important resource in coping with traumas. Such ruminations are related to negative thinking, counterproductive behaviours, and poor problem-solving in intra- and interpersonal relationships.

Studies (Ertan et al., 2020; Graham-Harrison et al., 2020) reported that the domestic violence increased during the lockdowns. For example, the Minister of Interior in France reported a 30\% increase in cases of domestic violence across the country and a 36\% increase in Paris alone since 17 March 2020; calls on emergency helplines in Spain reported an increase of domestic violence by $20 \%$; $30 \%$ in Cyprus; $25 \%$ in the UK; and 40\% - 50\% in Brazil. Increases in the need for emergency shelter and reports of domestic violence have also been observed in Australia, Canada, Germany, and the USA.

The COVID-19 pandemic as a traumatic event is an anxious process due to the risk of being infected. Such fear includes interrelated representations of feared stimuli (COVID-19 is around us), feared responses, and the meanings (contagion) associated with virus. A fear structure is activated by a fact that pandemic is a global worldwide danger thereby producing cognitive, behavioural, and physiological anxiety reactions. When a fear structure accurately represents dangerous situation in the world, it serves as a blueprint for effective actions which person is not able to control. Thus, a fear structure becomes maladaptive or pathological when 1) psychosocial and escape/avoidance responses are evoked by stimuli (evidence of Coronavirus), 2) excessive and easily triggered response elements interfere with adaptive behaviour ("The world is entirely dangerous", "I am completely incompetent"), and 3) stimulus and response elements are associated with threat meaning.

\section{Conclusion}

It is historically evident that the health emergencies such as epidemics, pandemics, wars, or natural catastrophes (i.e., earthquakes, severe flooding, bushfire) 
lead to detrimental and long-lasting psychological consequences. Epidemics and pandemics are associated with a wide range of psychopathological comorbidities at the global and individual levels, including anxiety, fears, depression, panic, and stress-related disorders. The COVID-19 pandemic is a disaster because of the exposure to a hazard that threatens personal, community and entire society safety, disrupts community and individual's self-structure, and results in personal and societal losses.

The COVID-19 pandemic has created, and still creates, complicated multiple stressors: the main abrupt changes in life; uncertainty about future; deterioration in livelihood; restrictions of social contacts and imposed quarantine or self-isolation; loss of loved ones; loneliness; and the threat of contracting COVID-19. It seems that the Coronavirus pandemic shares certain characteristics of the mass trauma (disaster) described in numerous studies.

However, the COVID-19 pandemic is specific as progressively emerging life threat of the entire world. The pandemic leads to insecurity, fears, pessimism, hopelessness, and uncertainty of the pandemic outcomes. Pandemic causes a significant loss of people's safety, freedom and basic psychobiological needs (i.e., relatedness and attachment, needs of competence, and autonomy), trustworthiness, sense of meanings, and the sense of control upon own self and activity.

During the pandemic, the social networks and one's psychic equilibrium are disrupted by strong lockdowns. People's chaotic behaviour is illustrated by hoarding and searching for some "reliable information about COVID-19". Individuals are severely aggravated by the different restrictive measures taken across the countries worldwide. Studies report that psychosocial impact seems to be even higher during quarantine measures.

Evidently, lockdown measures created a high-risk environment for the violent behaviour by increasing pandemic-related stressors and limiting to protective resources. Spending time in the same environment, and not having social contact with others, increases the risk of conflicts between the family members. Further, lockdown measures and pandemic-induced stress have important economic, social, and psychological consequences which decrease tolerance level of frustration and increase the risk of violent behaviour.

Physical distancing and lockdown measures may facilitate tension and reinforce the previously existing risk factors for violence. Studies (Ertan et al., 2020; Graham-Harrison et al., 2020) also report that quarantine and lockdowns lowered levels of life satisfaction, increased level of anxiety and depression, increase alcohol and substance abuse, and increase needs for the prescribed drugs to treat depression, anxiety, phobias, and stress-related conditions.

Currently published studies about the COVID-19 impact upon mental health have some important limitations, in particular the sampling technique. Most of them are web-based studies with recruitment of the general population, not specifically those who survive COVID-19 infection or had loved one ill, or deceased due to Coronavirus. The studies relied on voluntary participation through social 
networks, the surveys were based on self-report assessments, not on the interview-based measures, which limits the generalizability of the study findings.

It is possible that individuals with good health participated in the studies, while those individuals with the COVID-19 symptoms or higher stress due to Coronavirus were not adequately represented in the voluntary samples. However, this is quite acceptable considering limitations in social contacting those who are directly affected by the COVID-19. Considering the high rates of poor health outcomes in the general population, it is reasonable to assume that the studies among the individuals who directly experienced the life-threatening event (either as patients or family/acquaintances of the COVID-19 sufferers), will give a real picture of the Coronavirus impact upon mental health.

Certainly, the most stressful effect of the pandemic is upon those people which loved one deceased due to the COVID-19. Some of bereaved people did not have an opportunity to attend the funeral of the loved one either due to strictly imposed restrictions in gathering or because of being affected by the virus themselves. A significant majority of bereaved people fail to adjust to the loss of a loved one and develop persistent grief characterised by avoidance of reminders, difficulty to accepting death, bitterness, emotional numbness, and developed feeling that life is unfulfilling or meaningless.

Given the complexity and broad range of pandemic outcomes, it is likely that assessment of impact cannot be accomplished by measures only upon general population. At least, we shall admit that the COVID-19 pandemic is prolonged and still present life-threatening stressor. There is chronic apprehension of imminent doom, something terrible to happen, and any symbolic or actual sign of potential danger results in increased activity, agitation, fears, and pacing.

The intrusive symptoms, avoidance or constriction may persist with little change for many years after the pandemic. A pandemic is an extraordinary event, not because it occurs rarely, but rather because it overwhelms the ordinary adaptation to life. The common denominator of the pandemic is a feeling of helplessness, intense fear, loss of control, and threat of annihilation. Nothing less could be found among the healthcare workers who are dealing with the COVID-19 patients (Allan et al., 2020). For example, in survey of UK doctors in May 2020, $45 \%$ reported experiencing depression, anxiety, stress, burnout or other mental health problems during the pandemic (Green et al., 2021).

The experience of the pandemic is a shock to one's inner world with effects upon the one's self: hyperarousal, intrusive memories, dissociation, alterations in one's sense of the self-cohesion, pervasive sense of vigilance, distrust, feelings of emptiness, and depletion of self-awareness. Notably, the relatively high prevalence of mental health problems rate among the general population urges the need for a serious consideration of the psychiatric comorbidity of the COVID-19 pandemic once again. Of course, our greatest concern is for those individuals who develop chronic, enduring fear of dangerous world-individuals who likely will require medical intervention. 
The European Society for Traumatic Stress Studies (ESTSS) (Javakhishvili et al., 2020) recommended strategy to tackle mental health issues related to COVID19 focussing on: 1) trauma-informed policies; 2) capacity building; 3) collaborative research; and 4) knowledge-exchange. This strategy is not much different from the WHO's proposal (WHO, 2020) which reassures that the COVID-19 pandemic is a global mental health issue which requires united approach of the entire world.

The sole focus on physical health should be overcome by including impact upon mental health with the short- and long-term effects of the COVID-19 pandemic. This is in particular as studies (Hyland et al., 2020; Javakhishvili et al., 2020; Karatzias et al., 2020; Rossi et al., 2021) suggest that within a substantial proportion of the younger population the COVID-19 pandemic leads to the mental health problems (depression, anxiety, stress-related issues, withdrawal, aggressiveness), which require long-term strategy to deal with.

\section{Conflicts of Interest}

The author declares no conflicts of interest regarding the publication of this paper.

\section{References}

Allan, S. M., Bealey, J., Cushing, T., Sergi, G. et al. (2020). The Prevalence of Common and Stress-Related Health Disorder in Healthcare Workers Based in Pandemic-Affected Hospitals: A Rapid Systematic Review and Meat-Analysis. European Journal of Psychotraumatology, 11, Article No. 1810903. https://doi.org/10.1080/20008198.2020.1810903

APA (2013). Diagnostic and Statistical Manual of Mental Disorders (DSM-5). Washington DC: American Psychiatric Association.

Bialer, P. A., \& Wallack, J. J. (2000). Chapter 53. Human Immunodeficiency Virus and AIDS. In A. Stoudemire, B. S. Fogel, \& B. D. Greenberg (Eds.), Psychiatric Care of the Medical Patient (2nd ed., pp. 871-888). Oxford: Oxford University Press.

Brooks, S. K., Dunn, R., Amlot, R., Rubin, G. J., \& Greenberg, N. (2018). A systematic, Thematic Review of Social and Occupational Factors Associated with Psychological Outcomes in Healthcare Employers during an Infectious Disease Outbreak. Journal of Occupational and Environmental Medicine, 60, 248-257. https://doi.org/10.1097/JOM.0000000000001235

Cloitre, M., Hyland, P., Bisson, J. I., Brewin, C. R. et al. (2019). ICD-11 PTSD and Complex PTSD in the United States: A Population-Based Study. Journal of Traumatic Stress, 32, 833-842. https://doi.org/10.1002/jts.22454

Courtois, A. C., \& Ford, J. D. (2009). Treating Complex Traumatic Stress Disorder. New York: The Guilford Press.

Ertan, D., El-Hage, W., Thieree, S., Javelot, H., \& Hingray, C. (2020). COVID-19: Urgency for Distancing from Domestic Violence. European Journal of Psychotraumatology, 11, Article No. 1800245. https://doi.org/10.1080/20008198.2020.1800245

Ford, J. D., Courtois, A. C., van der Hart, O., \& Nijenhuis, E. R. S. (2005). Treatment of Complex Posttraumatic Self-Dysregulation. Journal of Traumatic Stress, 18, 437-447. https://doi.org/10.1002/jts.20051 
Graham-Harrison, E., Guilffrida, A., Smith, H., \& Ford, L. (2020). Lockdowns around the World Bring Rise in Domestic Violence. The Guardian.

Green, T., Harju-Steppanen, J., Adeniji, M. et al. (2021). Predictors and Rates of PTSD, Depression and Anxiety in UK Frontline Health and Social Care Workers during COVID-19. European Journal of Psychotraumatology, 12, Article No. 1882781. https://doi.org/10.1080/20008198.2021.1882781

Groves, M. S., \& Muskin, P. R. (2005). Chapter 5. Psychological Responses to illness. In J. L. Levenson (Ed.), Textbook of Psychosomatic Medicine (pp. 67-88). Washington DC: American Psychiatric Publishing.

Herman, J. (1992). Trauma and Recovery. New York: Basic Books.

Holmes, T. (1978). Life Situations, Emotions, and Disease. Psychosomatic Medicine, 19, 747-754. https://doi.org/10.1016/S0033-3182(78)70891-1

Hyland, P., Shelvin, M., McBride, O., Murphy, J. et al. (2020). Anxiety and Depression in the Republic of Ireland during the COVID-19 Pandemic. Acta Psychiatrica Scandinavica, 3, 249-256. https://doi.org/10.1111/acps.13219

Javakhishvili, D. J., Ardino, V., Bragesjo, M., Kazlauskas, E., Olff, M., \& Shafer, I. (2020). Trauma-Informed Responses in Addressing Public Mental Health Consequences of the COVID-19 Pandemic: Position Paper of the European Society for Traumatic Stress Studies (ESTSS). European Journal of Psychotraumatology, 11, Article No. 1780782. https://doi.org/10.1080/20008198.2020.1780782

Karatzias, T., Shevlin, M., Murphy, J., McBride, O. et al. (2020). Posttraumatic Stress Symptoms and Associated Comorbidity during the COVID-19 Pandemic in Ireland: A Population-Based Study. Journal of Traumatic Stress, 33, 365-370. https://doi.org/10.1002/jts.22565

Levenson, J. L., \& Schneider, R. K. (2005). Chapter 27. Infectious Diseases. In J. L. Levenson (Ed.), Textbook of Psychosomatic Medicine (pp. 577-598). Washington DC: American Psychiatric Publishing.

Rodriguez-Rey, R., Garrido-Hernansaiz, H., \& Colaldo, S. (2020). Psychological Impact of COVID-19 in Spain: Early Data Report. Psychological Trauma: Theory, Research, Practice, and Policy, 12, 550-552. https://doi.org/10.1037/tra0000943

Rossi, R., Socci, V., Talevi, D. et al. (2021). Trauma-Spectrum Symptoms among the Italian General Population in the Time of the COVID-19 Outbreak. European Journal of Psychotraumatology, 12, Article No. 1855888. https://doi.org/10.1080/20008198.2020.1855888

Stober, D. R., Schwartz, J. A. J. et al. (1997). Depression and HIV Disease: Prevalence, Correlates, and Treatment. Psychiatric Annals, 27, 372-377. https://doi.org/10.3928/0048-5713-19970501-14

Tucci, V., Moukaddam, N., Meadows, J. et al. (2017). The Forgotten Plague: Psychiatric Manifestations of Ebola, Zika, and Emerging Infectious Diseases. Journal of Global Infectious Diseases, 9, 151-156. https://doi.org/10.4103/jgid.jgid $66 \quad 17$

WHO (2018). International Statistical Classification of Disease and Related Health Problems (ICD-11). Geneva: World Health Organization. https://doi.org/10.1071/SH17086

WHO (2020). COVID-19 Briefings. Geneva: World Health Organization. https://www.who.int/

Zepinic, V. (2001). Chapter 14. Suicidal Risk in War-Related PTSD. In B. Raphael, \& A. E. Malak (Eds.), Diversity and Mental Health Challenging Times (pp. 212-221). Sydney: TCMHC.

Zepinic, V. (2015). Persistence of "Survival Skills" as a Risk Factor for Suicide in Severely 
Traumatised Individuals. International Journal of Emergency Mental Health and Human Resilience, 17, 552-558.

Zepinic, V. (2017). Disintegration of the Self-Structure Caused by Severe Trauma. Psychology and Behavioural Sciences, 5, 83-92. https://doi.org/10.11648/j.pbs.20160504.12

Zepinic, V. (2019). The Self and Complex Trauma (2nd ed.). London: Austin Macauley Publishing.

Zepinic, V. (2021). Complex Trauma Syndrome: Concept and Therapy. London: Austin Macauley Publishing. (In Print) 